\title{
Self-reported Taste and Smell Disorders in Patients with COVID-19: Distinct Features in China*
}

\author{
Jia SONG ${ }^{\dagger}$, Yi-ke DENG ${ }^{1 \dagger}$, Hai WANG ${ }^{1 \dagger}$, Zhi-chao WANG ${ }^{1}$, Bo LIAO ${ }^{1}$, Jin MA ${ }^{1}$, Chao HE ${ }^{1}$, Li PAN ${ }^{1}$, Yang LIU1 ${ }^{1}$ Isam Alobid ${ }^{2}$, \\ De-yun $\mathrm{WANG}^{3}$, Ming ZENG ${ }^{1 \#}$, Joaquim Mullol ${ }^{2 \#}$, Zheng LIU ${ }^{1 \#}$ \\ ${ }^{1}$ Department of Otolaryngology-Head and Neck Surgery, Tongji Hospital, Tongji Medical College, Huazhong University of \\ Science and Technology, Wuhan 430030, China \\ ${ }^{2}$ Rhinology Unit and Smell Clinic, ENT Department, Hospital Clinic, IDIBAPS, Universitat de Barcelona, CIBERES, Barcelona \\ 08036, Spain \\ ${ }^{3}$ Department of Otolaryngology, Yong Loo Lin School of Medicine, National University of Singapore 699010, Singapore
}

(C) Huazhong University of Science and Technology 2021

\begin{abstract}
Summary: Last December 2019, a cluster of viral pneumonia cases identified as coronavirus disease 2019 (COVID-19) was reported in Wuhan, China. We aimed to explore the frequencies of nasal symptoms in patients with COVID-19, including loss of smell and taste, as well as their presentation as the first symptom of the disease and their association with the severity of COVID-19. In this retrospective study, 1206 laboratory-confirmed COVID-19 patients were included and followed up by telephone one month after discharged from Tongji Hospital, Wuhan. Demographic data, laboratory values, comorbidities, symptoms, and numerical rating scale scores $(0-10)$ of nasal symptoms were extracted from the hospital medical records, and confirmed or reevaluated by the telephone follow-up. From patients $(n=1172)$ completing follow-up, $199(17 \%)$ subjects had severe COVID-19 and 342 (29.2\%) reported nasal symptoms. 20.6\% COVID-19 patients had loss of taste (median score $=6$ ), while $11.4 \%$ had loss of smell (median score $=5$ ). Loss of taste scores, but not loss of smell scores, were significantly increased in severe vs. nonsevere COVID-19 patients. Interleukin (IL)-6 and lactose dehydrogenase (LDH) serum levels were positively correlated with loss of taste scores. About $80 \%$ of COVID-19 patients recovered from smell and taste dysfunction in 2 weeks. In this cohort, only 1 out of 10 hospital admitted patients had loss of smell while 1 out of 5 reported loss of taste which was associated to severity of COVID-19. Most patients recovered smell and taste dysfunctions in 2 weeks.
\end{abstract}

Key words: taste; smell; coronavirus disease 2019; severe acute respiratory syndrome coronavirus 2

Coronavirus disease 2019 (COVID-19) has spread rapidly all over the world ${ }^{[1-3]}$. The diagnosis of the COVID-19 is based on clinical manifestations, contact history report, chest computed tomography (CT) and positive result of nucleic acid test ${ }^{[1]}$. Due to the uncertainty of contact history and the rush to

Jia SONG, E-mail: songjia-rhinology@outlook.com; Yi-ke DENG, E-mail: 1012934143@qq.com; Hai WANG, E-mail: 814899096@qq.com

${ }^{\dagger}$ The authors contributed equally to this work.

"Corresponding authors, Zheng LIU, E-mail: zhengliuent@, hotmail.com; Joaquim Mullol, E-mail: jmullol@clinic.cat; Ming ZENG, E-mail: zmsx77@163.com

${ }^{*}$ This study was supported by grants from the Natural Science Foundation of Hubei Province of China (No. 2018CFB602), National Key R\&D Program of China (No. 2018YFC0116800), and National Natural Science Foundation of China (Nos: 81630024, 81920108011, and 81900925).

Electronic supplementary material The online version of this article (https://doi.org/10.1007/s11596-021-2312-7) contains supplementary material, which is available to authorized users. hospitals that could run out of the essential equipment for test during the world-wide pandemic, efforts to identify additional diagnostic or prognostic symptoms of COVID-19 have significant value in mitigating transmission.

The clinical spectrum of COVID-19 ranges from asymptomatic to severe ill cases ${ }^{[1-3]}$. The most common symptom of COVID-19 is fever, and other common systemic symptoms include dyspnea, cough, nausea and vomiting, etc ${ }^{[1-3]}$. In addition, olfactory and taste disorders have been recently noted in patients with COVID-19. An early study conducted in Italy reported $33.9 \%$ of hospitalized COVID-19 patients showed at least one of taste or olfactory disorders ${ }^{[2]}$. Later studies in Europe reported that about $75 \%-85 \%$ COVID-19 patients had olfactory dysfunction and about $70 \%-$ $88 \%$ patients had gustatory dysfunctions ${ }^{[3]}$. Very recently, similar prevalence of smell and taste disorders has been found in COVID-19 patients in USA ${ }^{[4]}$. It is indicated that smell or taste change may be a strong predictor for a COVID-19 positive test result and 
serve as an early alerting symptom for COVID-19 ${ }^{[5]}$. However, an early study based on analyzing electronic medical records of 214 patients with COVID-19 in Wuhan, China reported that the proportion of patients complaining of loss of taste and loss of smell was only $5.6 \%$ and $5.1 \%$, respectively ${ }^{[6]}$, significantly lower than that reported in Europe and USA. One potential reason for the low rates in China may be related to incomplete medical records of COVID-19 patients under actual emergency situation, which underestimated the incidences of upper airway tract manifestations. Nevertheless, it is also possible that there are different responses to severe acute respiratory syndrome coronavirus 2 (SARS-CoV-2) infection in people with distinct ethnic/culture background. Therefore, more accurate evaluation of upper airway tract manifestations in COVID-19 patients should be conducted to figure out the clinical importance of smell and taste dysfunction in the early diagnosis of COVID-19 for Chinese and to elucidate whether there are different clinical manifestations between patients with distinct ethnic/culture background. Moreover, several important questions remain to be answered. How severe are the upper airway tract symptoms in patients with COVID-19? Is there any correlation between olfactory and taste disorders and other nasal symptoms such as nasal obstruction? Will the severity of olfactory or taste disorder be associated with the severity of COVID-19? Will there be a full recovery of olfactory or taste disorder and how long it will take?

In this retrospective study, we investigated the COVID-19 patients discharged from Tongji Hospital, the largest designated hospital to treat patients with COVID-19, in Wuhan. By integrating medical record analysis and reevaluation of upper airway symptoms via the telephone follow-up, we aimed to explore the frequencies of nasal symptoms in patients with COVID-19, including loss of smell and taste, as well as their presentation as the first symptom of the disease and their association with the severity of COVID-19.

\section{MATERIALS AND METHODS}

\subsection{Study Participants}

A single center, retrospective cohort study was conducted in Wuhan, China. We obtained the electronic medical records for discharged COVID-19 patients between January 27, 2020 and March 10, 2020, who were initially admitted to Tongji Hospital. The diagnosis was made on the basis of guidance for diagnosis and management of COVID-19 released by $\mathrm{WHO}^{[7]}$. A laboratory-confirmed case of COVID-19 was defined as having positive result on real-time reverse-transcriptase-polymerase-chain-reaction assay of nasal and pharyngeal swab specimens. Only laboratory-confirmed cases were included in the study.
All patients were followed up by telephone on the 30th $( \pm 2)$ day after discharge. The study was approved by Tongji Hospital Research Ethics Committee.

\subsection{Clinical Characteristic, Laboratory Assessment,} and Telephone Follow-up

The degree of severity of COVID-19 was defined as severe and non-severe at the time of admission using the American Thoracic Society guidelines for community-acquired pneumonia ${ }^{[8]}$. The information of demographic characteristics, systemic major symptoms, and major commodities related to COVID-19 were extracted from electronic medical records. In addition, the results of laboratory assessments on admission were also collected from electronic medical records. All laboratory testing was performed according to the clinical care needs of the patients. Laboratory assessments consisted of a complete blood routine, blood biochemistry, coagulation function, infection biomarkers and immune function. All data were entered into a computerized database and cross-checked.

The airway comorbidities and nasal symptoms, the date of symptomatic onset, and numerical rating scale scores and duration days of symptoms were obtained based on the hospital medical records and were confirmed and reevaluated by the telephone followup. The severity of upper respiratory tract symptoms was scored by patients on a numerical rating scale of $0-10$, with 0 being "no complaint whatsoever" and 10 being "the worst imaginable complaint" $"[9,10]$. Six major symptoms of upper respiratory tract were focused on: nasal obstruction, rhinorrhea, nasal itching, sneezing, loss of smell, and loss of taste. The severity of loss of smell and loss of taste was defined as follows: mild $=$ score $0-3$; moderate $=$ score $4-7$; severe $=$ score $8-10^{[9,10]}$. The difference between the symptom of loss of smell and loss of taste was explained to the patients very carefully during telephone follow-up according to previous studies ${ }^{[11]}$. The detailed questionnaire is shown in this article's Online Supplement.

\subsection{Statistical Analysis}

For continuous variables, Kruskal-Wallis $H$ test was used to assess intergroup variability and MannWhitney $U$ 2-tailed test was used for between-group comparison. Chi-square test was applied to compare the difference in proportions between groups. Spearman test was used for correlation analysis. Difference was considered to be statistically significant if a $P$ value was less than 0.05 . These statistical analyses were performed by an IBM SPSS 22.0 package (SPSS Inc, USA).

\section{RESULTS}

\subsection{Demographic and Clinical Characteristics}

Totally 1206 patients were enrolled and 1172 of them completed questionnaires. The follow-up rate was 
97.2\%. Reasons of the lost cases included: refusal to answer questions for personal reasons $(n=23)$; unable to provide accurate information $(n=6)$; not answering phone calls $(n=5)$. The demographic and clinical characteristics of 1172 patients are shown in table 1 . The median age was 61 years (IQR, 48-68), and 577 $(49.2 \%)$ were men. The severe cases accounted for $17 \%(199 / 1172)$.

Of the 1172 cases, 399 (25.1\%) had at least one comorbidity, and $17.3 \%$ had one or more respiratory comorbidities, including allergic rhinitis (AR, 9.8\%), chronic rhinosinusitis (CRS, 6.1\%), asthma (2.5\%) and chronic obstructive pulmonary disease (COPD, $0.9 \%)$. The frequency of patients with at least one nasal symptom was up to $29.2 \%$, including nasal obstruction (8.6\%; median score, 3), rhinorrhea (10.3\%; median score, 3$)$, nasal itching (4.9\%; median score, 2), sneezing (11.0\%; median score, 2), loss of smell $(11.4 \%$; median score, 5$)$, and loss of taste (20.6\%; median score, 6$)$. The incidence of symptom reported as the first onset symptom was $<1 \%$ for each individual nasal symptom, including loss of smell and taste. No significant difference in frequency of patients with loss of smell or taste disorder was found between severe and non-severe COVID-19 cases (fig. 1A). The scores of loss of taste but not smell were significantly higher in the patients with severe than in non-severe COVID-19 [7 (5-9) vs. 6 (4-8); $P=0.03$ )] (fig. 1B). No significant difference in frequency or score for the other nasal symptoms was found between severe and non-severe disease.

Senses of smell and taste are determined by the chemosensory system of the upper respiratory tract, which could be impacted by the nasal dysfunction ${ }^{[11]}$. Hence, we analyzed the relations between the scores of loss of taste and smell and other nasal symptoms. We failed to find any correlation between loss of taste or loss of smell scores and scores of the other nasal symptoms (fig. E1 in the Online Supplement). However, loss of taste showed mild positive correlation with loss of sense of smell $(\rho=0.25, P<0.01)$ (fig. E1 in the Online Supplement).

Given the possibility that some patients might not well distinguish the taste and smell disorder ${ }^{[11]}$, we compared the differences among the patients only with one smell or taste disorders, the patients with both smell and taste disorders, and the patients without any of these two symptoms. We found that the patients without loss of smell and taste were significantly elder than the patients in the other two groups [62 (48-69) years vs. 59 (46-67) years, 57.5 (42.75-66) years, $P=0.03)$ ]. No difference in other clinical characteristics and laboratory measurements was found among three groups. The data are shown in table E1 in the Online Supplement.

\subsection{Recovery of Olfactory and Taste Function}

We found that $82.1 \%(110 / 134)$ of patients with loss of sense of smell and 95.5\% (231/242) of patients with loss of taste recovered in one month after discharge. The symptomatic duration days showed no
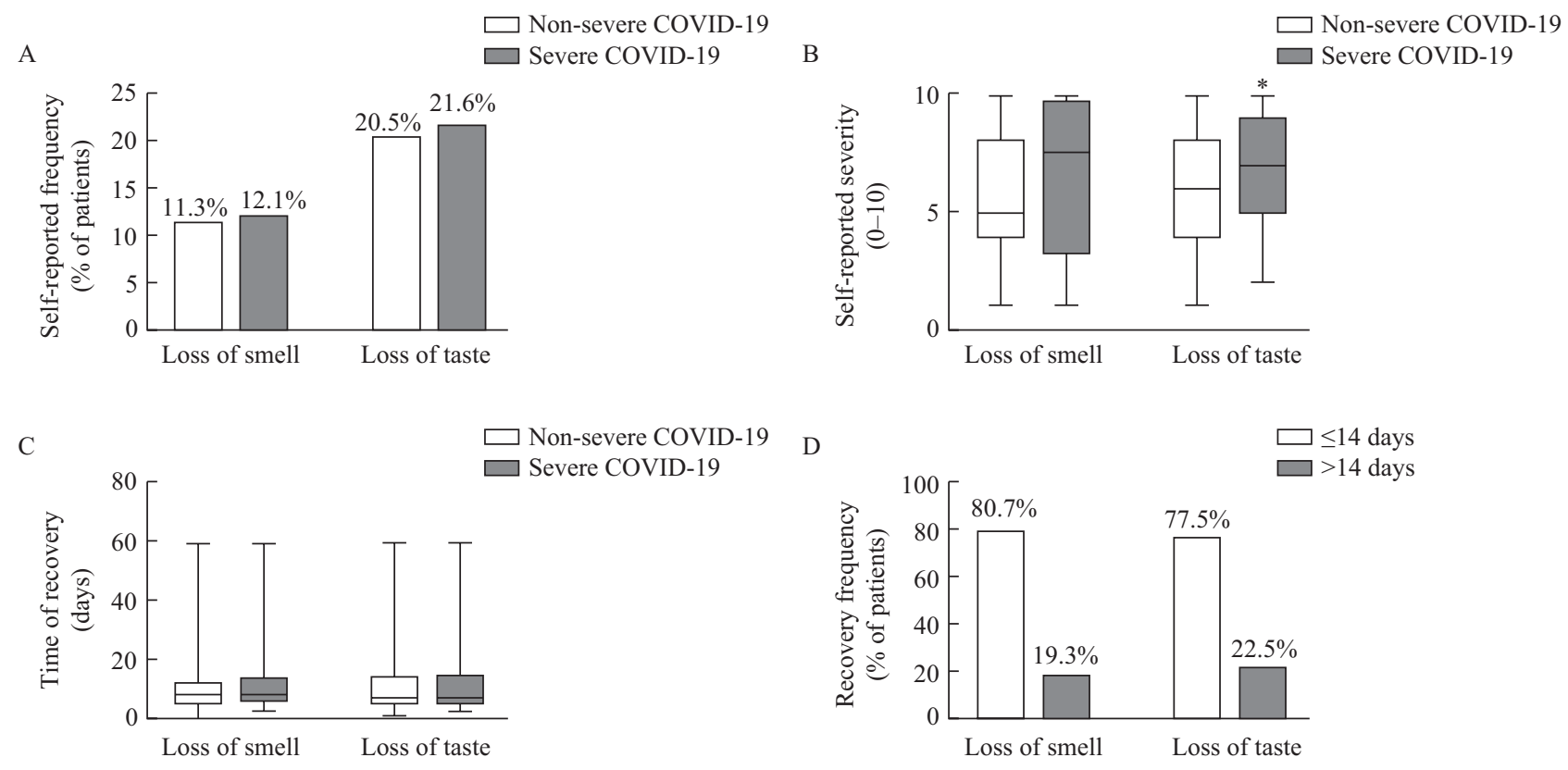

Fig. 1 The impact of coronavirus disease 2019 (COVID-19) on smell and taste

A: the prevalence of self-reported loss of smell and taste in severe and non-severe COVID-19 patients. The frequencies are indicated on the top of the columns. B: the severity of self-reported loss of smell and taste in severe and non-severe COVID-19 patients. Severity of self-reported loss of smell and taste symptom was scored by patients on a numerical rating scale of $0-10$, with 0 being "no complaint whatsoever" and 10 being "the worst imaginable complaint. C: the recovery time of self-reported smell and state dysfunction in severe and non-severe COVID-19 patients. D: the pattern of recovery time for patients with selfreported loss of smell and taste. The frequencies are indicated on the top of columns. ${ }^{*} P<0.05$ vs. non-severe COVID-19 
significant difference between the patients with loss of smell and taste [8 (6-13.25) vs. $7(5-14)$ days, $P=0.52)$ (fig. 1C). Most of them recovered in 14 days after onset of symptom (fig. 1D). No significant difference in recovery frequency of smell function was found between severe $(83.3 \%)$ and non-severe cases $(81.8 \%)$ $(P=0.99)$ (table 1$)$. As to taste disorder, 95.3\% patients with severe COVID-19 and $95.5 \%$ patients with non-severe COVID-19 recovered, and no significant difference was showed neither $(P=0.99)$ (table 1). Due to the limited number of patients with un-recovered symptoms, we did not compare the differences between recovered and un-recovered patients with loss of smell or loss of taste.

\subsection{Clinical Characteristics of COVID-19 Patients} with Different Severity of Taste and Smell Disorder

Since the taste disorder is one of the most common upper respiratory tract symptoms and shows a positive correlation with the symptom of loss of smell, we subsequently compared the differences among the COVID-19 patients with different severity of loss of taste (table 2) and loss of smell (table 3). First, we divided 242 cases of COVID-19 with loss of taste into mild (score, $1-3 ; 19.0 \%$ ), moderate (score, $4-7 ; 48.8 \%$ ) and severe (score, $8-10 ; 32.2 \%$ ) groups according to symptom scores, similar to the visual analogue scale system of nasal symptoms ${ }^{[10]}$. More COVID-19 cases with severe illness were found in the severe loss of

Table 1 Demographic and clinical characteristics of 1172 etiologically confirmed patients

\begin{tabular}{|c|c|c|c|c|}
\hline Characteristics & Total patients & Non-severe & Severe & $P$ value \\
\hline Subject, $n(\%)$ & $1172(100)$ & $973(83.0)$ & $199(17.0)$ & - \\
\hline Gender, male, $n(\%)$ & $577(49.2)$ & $480(49.3)$ & $97(48.7)$ & 0.94 \\
\hline Age, years, median (IQR) & $61(48,68)$ & $60(46,68)$ & $64(53,70)$ & $<0.01$ \\
\hline Systemic signs and symptoms, $n(\%)$ & - & - & - & - \\
\hline Fever & $921(78.6)$ & $758(77.9)$ & $163(81.9)$ & 0.21 \\
\hline Cough & $767(65.4)$ & $625(63.2)$ & $142(71.4)$ & 0.06 \\
\hline Myalgia & $172(14.7)$ & $136(14.0)$ & $36(18.1)$ & 0.13 \\
\hline Fatigue & $285(24.3)$ & $226(23.2)$ & $59(29.7)$ & 0.05 \\
\hline Anorexia & $274(23.4)$ & $214(22.0)$ & $60(30.2)$ & 0.01 \\
\hline Confusion & $6(0.5)$ & $3(0.3)$ & $3(1.5)$ & 0.03 \\
\hline Dizziness & $52(4.4)$ & $41(4.2)$ & $11(5.5)$ & 0.41 \\
\hline Any airway comorbidity, $n(\%)$ & $203(17.3)$ & $179(18.4)$ & $24(12.1)$ & 0.03 \\
\hline $\mathrm{AR}$ & $115(9.8)$ & $99(10.2)$ & $16(8.0)$ & 0.43 \\
\hline CRS & $72(6.1)$ & $64(6.6)$ & $8(4.5)$ & 0.34 \\
\hline Asthma & $29(2.5)$ & $25(2.6)$ & $4(2.0)$ & 0.81 \\
\hline COPD & $10(0.9)$ & $10(1.0)$ & $0(0)$ & 0.23 \\
\hline Any nasal symptom, $n(\%)$ & $342(29.2)$ & $275(28.3)$ & $67(33.7)$ & 0.15 \\
\hline Nasal obstruction, $n(\%)$ & $101(8.6)$ & $82(8.4)$ & $19(9.5)$ & 0.58 \\
\hline Score, median (IQR) & $3(2,4)$ & $3(2,4)$ & $3(2,5)$ & 0.88 \\
\hline As first symptom, $n(\%)$ & $2(0.2)$ & $1(0.1)$ & $1(0.5)$ & 0.31 \\
\hline Rhinorrhea, $n(\%)$ & $120(10.3)$ & $100(10.3)$ & $20(10.1)$ & 0.99 \\
\hline Score, median (IQR) & $3(2,4)$ & $3(2,4)$ & $2.5(1.25,3.75)$ & 0.37 \\
\hline As first symptom, $n(\%)$ & $9(0.8)$ & $8(0.8)$ & $1(0.5)$ & 0.99 \\
\hline Nasal itching, $n(\%)$ & $57(4.9)$ & $45(4.6)$ & $12(6.0)$ & 0.37 \\
\hline Score, median (IQR) & $2(1.5,3)$ & $2(2,3.5)$ & $2(1,2)$ & 0.08 \\
\hline As first symptom, $n(\%)$ & $1(0.1)$ & $1(0.1)$ & $0(0)$ & 0.99 \\
\hline Sneezing, $n(\%)$ & $129(11.0)$ & $104(10.7)$ & $25(12.6)$ & 0.46 \\
\hline Score, median (IQR) & $2(1,3)$ & $2(1,3)$ & $2(1,2.5)$ & 0.77 \\
\hline As first symptom, $n(\%)$ & $1(0.1)$ & $1(0.1)$ & $0(0)$ & 0.99 \\
\hline Loss of smell, $n(\%)$ & $134(11.4)$ & $110(11.3)$ & $24(12.1)$ & 0.71 \\
\hline Score, median (IQR) & $5(4,9)$ & $5(4,8)$ & $7.5(3.25,10)$ & 0.37 \\
\hline As first symptom, $n(\%)$ & $3(0.3)$ & $3(0.3)$ & $0(0)$ & 0.99 \\
\hline Loss of smell recovered, $n(\%)$ & $110(82.1 \%)$ & $90(81.8 \%)$ & $20(83.3 \%)$ & 0.99 \\
\hline Recovery time, days, median (IQR) & $8(6,13.25)$ & $8(5,12.25)$ & $8(6.25,14)$ & 0.67 \\
\hline Loss of taste, $n(\%)$ & $242(20.6)$ & $199(20.5)$ & $43(21.6)$ & 0.70 \\
\hline Score, median (IQR) & $6(4,8)$ & $6(4,8)$ & $7(5,9)$ & 0.03 \\
\hline As first symptom, $n(\%)$ & $5(0.4)$ & $4(0.4)$ & $1(0.5)$ & 0.99 \\
\hline Loss of taste recovered, $n(\%)$ & $231(95.5 \%)$ & $190(95.5 \%)$ & $41(95.3 \%)$ & 0.99 \\
\hline Recovery time, days, median (IQR) & $7(5,14)$ & $7(5,14)$ & $7(5,14.5)$ & 0.76 \\
\hline
\end{tabular}

IQR, interquartile range; AR, allergic rhinitis; CRS, chronic rhinosinusitis; COPD, chronic obstructive pulmonary disease. Data are presented as medians and interquartile ranges (IQR) for continuous variables and number with percentage for categorical variables. $P$ values were calculated from Mann-Witney $U$ 2-tailed test, $\chi^{2}$ test or Fisher's exact test. 
Table 2 The comparison of 242 patients with different severity of loss of taste

\begin{tabular}{|c|c|c|c|c|}
\hline Characteristics & Mild (0-3) & Moderate (4-7) & Severe $(8-10)$ & $P$ value \\
\hline Subject, $n(\%)$ & $46(19.0)$ & $118(48.8)$ & $78(32.2)$ & - \\
\hline Gender, male, $n(\%)$ & $21(45.7)$ & $59(50.0)$ & $39(50.0)$ & 0.16 \\
\hline Age, years, median (IQR) & $55(42,67)$ & $58.5(43.75,67)$ & $58.5(48.5,66)$ & 0.88 \\
\hline Severe cases, $n(\%)$ & $7(15.2)$ & $15(12.7)$ & $21(26.9)^{\#}$ & 0.03 \\
\hline Loss of taste recovered, $n(\%)$ & $45(97.8)$ & $110(93.2)$ & $76(97.4)$ & 0.26 \\
\hline Recovery time, days, median (IQR) & $5(3.5,8)$ & $8(6,13.25)^{* *}$ & $10(5,15)^{* *}$ & $<0.01$ \\
\hline Any airway comorbidity, $n(\%)$ & $6(13.0)$ & $26(22.0)$ & $13(16.7)$ & 0.36 \\
\hline AR & $2(4.3)$ & $12(10.2)$ & $9(11.5)$ & 0.39 \\
\hline CRS & $3(6.5)$ & $10(5.6)$ & $4(5.1)$ & 0.66 \\
\hline Asthma & $1(2.2)$ & $3(2.5)$ & $2(2.6)$ & 0.99 \\
\hline COPD & $0(0)$ & $3(2.5)$ & $0(0)$ & 0.20 \\
\hline Systemic signs and symptoms, $n(\%)$ & - & - & - & - \\
\hline Fever & $37(80.4)$ & $101(85.6)$ & $66(84.6)$ & 0.71 \\
\hline Cough & $29(61.7)$ & $78(66.1)$ & $53(70.0)$ & 0.77 \\
\hline Myalgia & $7(15.2)$ & $18(15.3)$ & $10(12.8)$ & 0.88 \\
\hline Fatigue & $12(26.1)$ & $34(28.8)$ & $18(23.1)$ & 0.80 \\
\hline Anorexia & $12(26.1)$ & $30(25.4)$ & $18(23.1)$ & 0.91 \\
\hline Confusion & $1(2.2)$ & $0(0)$ & $0(0)$ & 0.12 \\
\hline Dizziness & $2(4.3)$ & $3(2.5)$ & $4(5.1)$ & 0.63 \\
\hline Laboratory results, median (IQR) & - & - & - & - \\
\hline Blood routine, subject, $n$ & 46 & 118 & 78 & - \\
\hline White blood cell count, $\times 10^{9} / \mathrm{L}$ & $5.7(3.8,6.8)$ & $5.7(4.5,7.5)$ & $5.8(4.6,7.4)$ & 0.48 \\
\hline Neutrophil count, $\times 10^{9} / \mathrm{L}$ & $3.5(2.6,5.6)$ & $3.8(2.7,5.5)$ & $4.0(2.8,5.5)$ & 0.96 \\
\hline Neutrophil percentage & $66.8(57.0,75.0)$ & $64.1(57.3,72.6)$ & $66.8(58.7,73.0)$ & 0.88 \\
\hline Lymphocyte count, $\times 10^{9} / \mathrm{L}$ & $1.1(0.8,2.0)$ & $1.3(0.9,1.7)$ & $1.2(0.9,1.6)$ & 0.80 \\
\hline Lymphocyte percentage & $23.1(18,31.8)$ & $22.8(16,30.4)$ & $21.7(15.4,28.7)$ & 0.53 \\
\hline Monocyte count, $\times 10^{9} / \mathrm{L}$ & $0.5(0.4,0.6)$ & $0.5(0.4,0.6)$ & $0.5(0.4,0.7)$ & 0.33 \\
\hline Monocyte percentage & $8.5(6.1,11.4)$ & $9.0(7.0,11.1)$ & $9.5(7.8,10.6)$ & 0.33 \\
\hline Eosinophil count, $\times 10^{9} / \mathrm{L}$ & $0.03(0,0.08)$ & $0.06(0.01,0.11)$ & $0.04(0,0.09)$ & 0.14 \\
\hline Eosinophil percentage & $0.4(0,1.2)$ & $0.95(0.2,1.8)$ & $0.6(0,1.7)$ & 0.07 \\
\hline Platelet count, $\times 10^{9} / \mathrm{L}$ & $227(187.8,312)$ & $252(195,314)$ & $260(190.8,328)$ & 0.27 \\
\hline Hemoglobin, g/L & $128(114.8,137.5)$ & $129(113.8,137)$ & $129(120,141.3)$ & 0.36 \\
\hline Liver and renal function test, subject, $n$ & 46 & 118 & 78 & - \\
\hline Alanine aminotransferase, $\mathrm{U} / \mathrm{L}$ & $22.5(13,35.3)$ & $24.5(16.8,44)$ & $29(17,43)$ & 0.15 \\
\hline Aspartate aminotransferase, $\mathrm{U} / \mathrm{L}$ & $24.5(19,35)$ & $21(18,34)$ & $26.5(19,37.5)$ & 0.30 \\
\hline Albumin, g/L & $37.2(33.1,41.8)$ & $36.7(32.7,40.2)$ & $35.1(32.3,37.6)$ & 0.08 \\
\hline Globulin, g/L & $32.2(29.3,34.9)$ & $32.4(22.2,35.8)$ & $33.2(29.8,36.5)$ & 0.48 \\
\hline Alkaline phosphatase, $\mathrm{U} / \mathrm{L}$ & $58(50,72.75)$ & $63(55,80.25)$ & $60(52.25,81.5)$ & 0.15 \\
\hline Total bilirubin, $\mu \mathrm{mol} / \mathrm{L}$ & $8.4(5.6,13)$ & $8.4(6.4,11.7)$ & $8.8(7,11.4)$ & 0.83 \\
\hline Lactose dehydrogenase, U/L & $229.5(179.5,305.8)$ & $239.5(197,300)$ & $276.5(224.5,347.5)^{* \#}$ & 0.04 \\
\hline Urea, $\mathrm{mmol} / \mathrm{L}$ & $4.3(3.2,5.7)$ & $4.1(3.3,5.2)$ & $3.75(3.2,4.83)$ & 0.88 \\
\hline Creatinine, $\mu \mathrm{mol} / \mathrm{L}$ & $67.5(59,77)$ & $69(55,82)$ & $65.5(56,81)$ & 0.98 \\
\hline Urine acid, $\mu \mathrm{mol} / \mathrm{L}$ & $276.9(222.8,338.2)$ & $248(190.7,327.5)$ & $241.4(201.3,293.8)$ & 0.13 \\
\hline Glomerular filtration, $\mathrm{mL} / \mathrm{min} / 1.73 \mathrm{~m}^{2}$ & $93.3(82.9,109.5)$ & $95.7(83.9,107.9)$ & $94.2(83.75,106.1)$ & 0.91 \\
\hline Electrolytes, subject, $n$ & 46 & 118 & 78 & - \\
\hline $\mathrm{K}^{+}, \mathrm{mmol} / \mathrm{L}$ & $4.2(3.9,4.6)$ & $4.2(3.9,4.5)$ & $4.1(3.8,4.4)$ & 0.15 \\
\hline $\mathrm{Na}^{+}, \mathrm{mmol} / \mathrm{L}$ & $139.4(137.8,141.6)$ & $140.5(138.2,142)$ & $139.5(137.7,141.3)$ & 0.31 \\
\hline $\mathrm{Cl}^{-}, \mathrm{mmol} / \mathrm{L}$ & $100.8(99,103)$ & $101.6(99.1,103.7)$ & $100.8(98.1,103.1)$ & 0.34 \\
\hline $\mathrm{Ca}^{2+}, \mathrm{mmol} / \mathrm{L}$ & $2.14(2.04,2.24)$ & $2.16(2.08,2.25)$ & $2.15(2.09,2.22)$ & 0.64 \\
\hline $\mathrm{ESR}, \mathrm{mm} / \mathrm{h}$ & $25(16.75,58)$ & $32(13,60)$ & $39(18,64.75)$ & 0.53 \\
\hline CRP, mg/L & $4.65(1.43,29.6)$ & $4.4(1.2,34)$ & $7.5(1.85,42.85)$ & 0.33 \\
\hline Cardiac troponin I, pg/mL & $2.5(1.9,6.9)$ & $2.9(1.9,7)$ & $3.5(1.9,6.9)$ & 0.81 \\
\hline Inflammatory mediators, subject, $n$ & 35 & 99 & 72 & - \\
\hline Ferritin, $\mu \mathrm{g} / \mathrm{L}$ & $467.7(183,633.5)$ & $531.1(294,827.8)$ & $576.7(310.8,1268)$ & 0.21 \\
\hline Interleukin-10, pg/mL & $5(5,5)$ & $5(5,5)$ & $5(5,5)$ & 0.25 \\
\hline Interleukin- $1 \beta, \mathrm{pg} / \mathrm{mL}$ & $5(5,5)$ & $5(5,5)$ & $5(5,5)$ & 0.55 \\
\hline Interleukin-2 receptor, $\mathrm{U} / \mathrm{mL}$ & $465(242,624)$ & $498(376,706)$ & $571(362.8,792.5)$ & 0.13 \\
\hline
\end{tabular}


(Continued from the last page)

\begin{tabular}{lcccc}
\hline Characteristics & Mild $(0-3)$ & Moderate $(4-7)$ & Severe $(8-10)$ & $P$ value \\
\hline Interleukin-6, pg/mL & $3.7(1.7,14.9)$ & $3.8(1.9,10.5)$ & $8.1(2.3,23.8)^{* *}$ & 0.03 \\
Interleukin-8, pg/mL & $6.5(5,10.5)$ & $10.4(5.5,19.5)^{*}$ & $9.9(5.3,18.7)^{*}$ & 0.04 \\
Tumor necrosis factor, pg/mL & $7(5.2,9.7)$ & $7(5.1,9.1)$ & $7.9(5.1,10.9)$ & 0.32 \\
Procalcitonin, $\mathrm{ng} / \mathrm{mL}$ & $0.05(0.03,0.07)$ & $0.05(0.03,0.08)$ & $0.05(0.02,0.09)$ & 0.98 \\
Immunoglobulins and complements, subject, $n$ & 11 & 43 & 25 & - \\
IgA, g/L & $2.2(1.6,3.1)$ & $2.2(1.8,2.6)$ & $2.2(1.6,2.5)$ & 0.97 \\
IgG, g/L & $10.6(9.2,11.6)$ & $11.1(9.6,13.2)$ & $11.6(10,13.6)$ & 0.51 \\
IgM, g/L & $0.79(0.64,0.96)$ & $1(0.79,1.3)$ & $0.91(0.65,1.4)$ & 0.12 \\
C3, g/L & $1(0.83,1.13)$ & $0.86(0.78,1)$ & $0.9(0.74,1.07)$ & 0.34 \\
C4, g/L & $0.24(0.22,0.36)$ & $0.22(0.18,0.28)$ & $0.26(0.2,0.3)$ & 0.16 \\
\hline
\end{tabular}

$\mathrm{IQR}$, interquartile range; AR, allergic rhinitis; CRS, chronic rhinosinusitis; COPD, chronic obstructive pulmonary disease; ERS, erythrocyte sedimentation rate; CRP, C-reactive protein; Ig, immunoglobulin; C, complement. Data are presented as medians and interquartile ranges (IQR) for continuous variables and number with percentage for categorical variables. $P$ values were calculated from Kruskal-Wallis, $\chi^{2}$ test or Fisher's exact test. ${ }^{*} P<0.05$ vs. mild, ${ }^{* *} P<0.01$ vs. mild, ${ }^{\#} P<0.05$ vs. moderate

Table 3 Comparison of 134 patients with different severity of loss of smell

\begin{tabular}{|c|c|c|c|c|}
\hline Characteristics & Mild (0-3) & Moderate (4-7) & Severe $(8-10)$ & $P$ value \\
\hline Subject, $n(\%)$ & $32(23.9)$ & $48(35.8)$ & $54(40.3)$ & - \\
\hline Gender, male, $n(\%)$ & $10(31.3)$ & $17(35.4)$ & $27(50.0)$ & 0.16 \\
\hline Age, years, median (IQR) & $58(49.25,63.75)$ & $57(46,68)$ & $57.5(42.25,65.25)$ & 0.82 \\
\hline Severe cases, $n(\%)$ & $6(18.8)$ & $6(12.5)$ & $12(22.2)$ & 0.44 \\
\hline Loss of taste recovered, $n(\%)$ & $28(87.5)$ & $36(75.0)$ & $46(85.2)$ & 0.37 \\
\hline Recovery time, days, median (IQR) & $7(5,14)$ & $7(5.25,10)$ & $10(7,17.5)$ & 0.08 \\
\hline Any airway comorbidity, $n(\%)$ & $8(25.0)$ & $10(20.8)$ & $9(16.7)$ & 0.64 \\
\hline AR & $2(6.3)$ & $5(10.4)$ & $7(13.0)$ & 0.61 \\
\hline CRS & $3(9.4)$ & $3(6.3)$ & $3(5.6)$ & 0.78 \\
\hline Asthma & $3(9.4)$ & $1(2.1)$ & $0(0)^{*}$ & 0.04 \\
\hline COPD & $0(0)$ & $1(2.1)$ & $0(0)$ & 0.45 \\
\hline Systemic signs and symptoms, $n(\%)$ & - & - & - & - \\
\hline Fever & $22(68.8)$ & $35(72.9)$ & $44(81.5)$ & 0.37 \\
\hline Cough & $23(71.9)$ & $32(66.7)$ & $88(65.7)$ & 0.58 \\
\hline Myalgia & $9(28.1)$ & $8(16.7)$ & $7(13.0)$ & 0.20 \\
\hline Fatigue & $13(40.6)$ & $12(25.0)$ & $11(20.4)$ & 0.11 \\
\hline Anorexia & $4(12.5)$ & $11(22.9)$ & $15(27.8)$ & 0.26 \\
\hline Confusion & $0(0)$ & $0(0)$ & $0(0)$ & - \\
\hline Dizziness & $2(6.3)$ & $3(6.3)$ & $4(7.4)$ & 0.97 \\
\hline Laboratory results, median (IQR) & - & - & - & - \\
\hline Blood routine, subject, $n$ & 32 & 48 & 54 & - \\
\hline White blood cell count, $\times 10^{9} / \mathrm{L}$ & $5.1(3.7,6.5)$ & $5.7(4.6,7.5)$ & $5.8(4.5,7.8)$ & 0.08 \\
\hline Neutrophil count, $\times 10^{9} / \mathrm{L}$ & $2.9(2.1,5.0)$ & $3.7(2.7,5.2)$ & $4.2(2.8,5.4)$ & 0.09 \\
\hline Neutrophil percentage & $61.2(53.0,73.1)$ & $64.1(57.1,74.8)$ & $70.2(58.6,74.7)$ & 0.33 \\
\hline Lymphocyte count, $\times 10^{9} / \mathrm{L}$ & $1.3(0.8,1.6)$ & $1.3(1.0,1.7)$ & $1.3(0.9,1.6)$ & 0.77 \\
\hline Lymphocyte percentage & $26.2(17.3,34.2)$ & $24.9(16.2,31.2)$ & $22.2(16.2,27.0)$ & 0.46 \\
\hline Monocyte count, $\times 10^{9} / \mathrm{L}$ & $0.4(0.4,0.5)$ & $0.5(0.4,0.6)$ & $0.5(0.4,0.7)$ & 0.09 \\
\hline Monocyte percentage & $9.0(7.2,10.9)$ & $8.9(7.0,10.1)$ & $8.5(6.5,10.5)$ & 0.67 \\
\hline Eosinophil count, $\times 10^{9} / \mathrm{L}$ & $0.03(0,0.1)$ & $0.05(0.02,0.08)$ & $0.04(0.01,0.09)$ & 0.61 \\
\hline Eosinophil percentage & $0.85(0,1.8)$ & $0.75(0.3,1.7)$ & $0.75(0.3,1.7)$ & 0.89 \\
\hline Platelet count, $\times 10^{9} / \mathrm{L}$ & $232.5(143.8,264.5)$ & $259.5(199.5,308)$ & $238(188.5,316)$ & 0.14 \\
\hline Hemoglobin, $g / L$ & $125.5(113,135.3)$ & $125.5(114,134.8)$ & $128.5(119.5,143.3)$ & 0.12 \\
\hline Liver and renal function test, subject, $n$ & 32 & 48 & 54 & - \\
\hline Alanine aminotransferase, $\mathrm{U} / \mathrm{L}$ & $18(13,49)$ & $20(12,31)^{*}$ & $29(17,43)$ & 0.04 \\
\hline Aspartate aminotransferase, U/L & $21.5(18.3,31.8)$ & $20.5(16,29)$ & $25(16,33.3)$ & 0.48 \\
\hline Albumin, g/L & $37.1(32.1,39.9)$ & $36.6(31.5,41)$ & $34.5(32.4,38.7)$ & 0.81 \\
\hline Globulin, g/L & $31.8(29.4,34.4)$ & $32.4(26.2,38.9)$ & $33.1(28.9,36)$ & 0.89 \\
\hline Alkaline phosphatase, U/L & $67.5(55,81.3)$ & $59(52,74.3)$ & $58.5(50,79.3)$ & 0.26 \\
\hline Total bilirubin, $\mu \mathrm{mol} / \mathrm{L}$ & $8.4(6.7,10.1)$ & $9.7(7.3,11.6)$ & $8.6(6.3,11.6)$ & 0.68 \\
\hline Lactose dehydrogenase, $\mathrm{U} / \mathrm{L}$ & $236(194,298.8)$ & $231.5(189.3,267.5)$ & $264.5(188.8,367.3)$ & 0.27 \\
\hline
\end{tabular}


(Continued from the last page)

\begin{tabular}{|c|c|c|c|c|}
\hline Characteristics & Mild (0-3) & Moderate (4-7) & Severe $(8-10)$ & $P$ value \\
\hline Urea, mmol/L & $3.8(3.4,4.4)$ & $4.5(3.3,5.2)$ & $4.0(3.3,5.5)$ & 0.47 \\
\hline Creatinine, $\mu \mathrm{mol} / \mathrm{L}$ & $51(37,59)$ & $62(53,78.3)$ & $64.5(55,80)$ & 0.43 \\
\hline Urine acid, $\mu \mathrm{mol} / \mathrm{L}$ & $242.9(182.1,302.5)$ & $271(223.5,327.1)$ & $233.5(198.5,282.3)$ & 0.11 \\
\hline Glomerular filtration, $\mathrm{mL} / \mathrm{min} / 1.73 \mathrm{~m}^{2}$ & $97.1(90.4,109.4)$ & $93.4(78.9,107.6)$ & $95.7(88.1,110.1)$ & 0.54 \\
\hline Electrolytes, subject, $n$ & 32 & 48 & 54 & - \\
\hline $\mathrm{K}^{+}, \mathrm{mmol} / \mathrm{L}$ & $4.0(3.8,4.2)$ & $4.1(3.7,4.3)$ & $4.1(3.7,4.5)$ & 0.56 \\
\hline $\mathrm{Na}^{+}, \mathrm{mmol} / \mathrm{L}$ & $140.9(137.6,142.3)$ & $139.8(138.1,141.8)$ & $139.4(137.7,140.9)$ & 0.17 \\
\hline $\mathrm{Cl}^{-}, \mathrm{mmol} / \mathrm{L}$ & $101.2(99.3,104.3)$ & $101.9(100.3,103.7)$ & $101(98,103.2)$ & 0.41 \\
\hline $\mathrm{Ca}^{++}, \mathrm{mmol} / \mathrm{L}$ & $2.15(2.04,2.26)$ & $2.20(2.08,2.27)$ & $2.15(2.08,2.23)$ & 0.46 \\
\hline Cardiac troponin I, pg/mL & $4.7(3,13.9)$ & $6.6(2.7,11.8)$ & $5(3.2,8.2)$ & 0.84 \\
\hline $\mathrm{ESR}, \mathrm{mm} / \mathrm{h}$ & $48(22,67)$ & $38.5(12,59.3)$ & $26.5(10.5,53)$ & 0.15 \\
\hline $\mathrm{CRP}, \mathrm{mg} / \mathrm{L}$ & $14.1(2,35.8)$ & $4(1.6,28.4)$ & $5.7(14.5,42.7)$ & 0.57 \\
\hline Inflammatory mediators, subject, $n$ & 26 & 45 & 48 & - \\
\hline Ferritin, $\mu \mathrm{g} / \mathrm{L}$ & $515.9(205.5,1304)$ & $426.7(228.1,645.3)$ & $663.1(328.3,1050)$ & 0.23 \\
\hline Interleukin-10, $\mathrm{pg} / \mathrm{mL}$ & $5(5,5.3)$ & $5(5,5)^{*}$ & $5(5,5.7)$ & 0.04 \\
\hline Interleukin- $1 \beta, \mathrm{pg} / \mathrm{mL}$ & $5(5,5)$ & $5(5,5)$ & $5(5,5)$ & 0.24 \\
\hline Interleukin-2 receptor, $\mathrm{U} / \mathrm{mL}$ & $523(344,751)$ & $428(279,688)$ & $554.5(322.3,711.3)$ & 0.47 \\
\hline Interleukin- $6, \mathrm{pg} / \mathrm{mL}$ & $3.8(2.1,13.6)$ & $3.7(1.5,8.6)$ & $4.1(1.6,20.0)$ & 0.46 \\
\hline Interleukin- $8, \mathrm{pg} / \mathrm{mL}$ & $7.7(5,16.2)$ & $9.1(5.2,12.3)$ & $10.2(5.1,21.8)$ & 0.42 \\
\hline Tumor necrosis factor, $\mathrm{pg} / \mathrm{mL}$ & $8.6(5.7,9.9)$ & $6(4.7,7.2)^{*}$ & $8.0(5.6,10.3)$ & $<0.01$ \\
\hline Procalcitonin, ng/mL & $0.05(0.04,0.09)$ & $0.04(0.02,0.07)$ & $0.05(0.03,0.09)$ & 0.35 \\
\hline Immunoglobulins and complements, subject, $n$ & 8 & 17 & 21 & - \\
\hline $\operatorname{IgA}, \mathrm{g} / \mathrm{L}$ & $2.1(1.9,2.3)$ & $2.2(1.2,2.8)$ & $2.3(1.6,2.8)$ & 0.83 \\
\hline $\mathrm{IgG}, \mathrm{g} / \mathrm{L}$ & $10.5(9.5,15.9)$ & $12.9(10.8,15.5)$ & $12.5(10.6,14.5)$ & 0.55 \\
\hline $\operatorname{IgM}, g / L$ & $0.94(0.81,1.2)$ & $1(0.82,1.34)$ & $0.86(0.67,1.21)$ & 0.56 \\
\hline $\mathrm{C} 3, \mathrm{~g} / \mathrm{L}$ & $0.91(0.72,1.1)$ & $0.89(0.72,0.97)$ & $0.92(0.78,1.05)$ & 0.68 \\
\hline $\mathrm{C} 4, \mathrm{~g} / \mathrm{L}$ & $0.23(0.19,0.24)$ & $0.18(0.11,0.21)$ & $0.21(0.16,0.33)$ & 0.94 \\
\hline
\end{tabular}

$\mathrm{IQR}$, interquartile range; AR, allergic rhinitis; CRS, chronic rhinosinusitis; COPD, chronic obstructive pulmonary disease; ERS, erythrocyte sedimentation rate; CRP, C-reactive protein; Ig, immunoglobulin; C, complement. Data are presented as medians and interquartile ranges (IQR) for continuous variables and number with percentage for categorical variables. $P$ values were calculated from Kruskal-Wallis, $\chi^{2}$ test or Fisher's exact test. ${ }^{*} P<0.05$ vs. mild

taste group than in the moderate loss of taste group ( $26.9 \%$ vs. $12.7 \%, P=0.03$ ). The symptom duration of loss of taste was significantly longer in moderate and severe taste dysfunction group than in the mild taste dysfunction group [8 days (6-13.25), 10 days (5-15) vs. 5 days (3.5-8), $P<0.01]$ (fig. 2 ). In addition, serum levels of interleukin-6 (IL-6) and lactate dehydrogenase $(\mathrm{LDH})$ were significantly increased in patients with

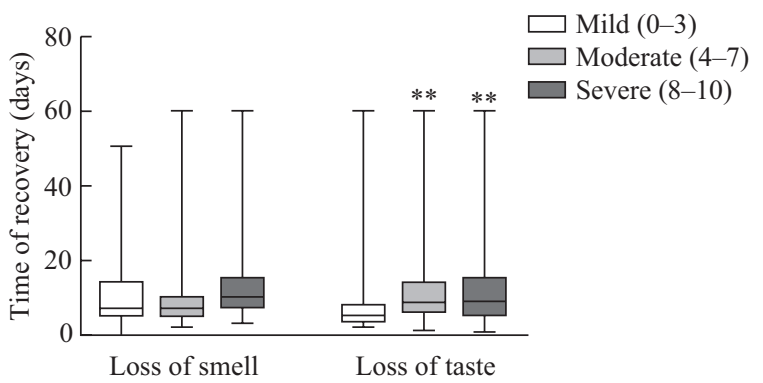

Fig. 2 The recovery time of patients with different severity of loss of smell and loss of taste

Severity of self-reported loss of smell and taste symptom was scored by patients on a numerical rating scale of $0-10$, with 0 being "no complaint whatsoever" and 10 being "the worst imaginable complaint". ${ }^{* *} P<0.01 \mathrm{vs}$. mild loss of taste severe vs. mild and moderate loss of taste groups (table 2). Levels of IL-8 were significantly increased in severe and moderate loss of taste group compared to mild group (table 2). The characteristics of the patients with different severity of taste disorder are shown in table 2. In addition, IL-6 and LDH showed a mild positive correlation to the symptom scores of loss of taste $(\rho=0.15, P=0.03 ; \rho=0.21, P<0.01$, respectively; fig. 3).

As to the comparison among different severity of loss of smell, we divided the 134 patients with loss of sense of smell into mild (score, 1-3, 23.9\%), moderate (score, 4-7; 35.8\%) and severe (score, 8-10; $40.3 \%$ ) groups. No significant difference in clinical and laboratory measurements was found among the patients with different severity (table 3 and fig. 2).

\section{DISCUSSION}

Overall, 17\% subjects were categorized as severe COVID-19, this percentage being similar with previous reports from China ${ }^{[1,6]}$. Most of the common systemic signs and symptoms of disease such as fever, fatigue, and anorexia, etc. reported in previous studies were also 

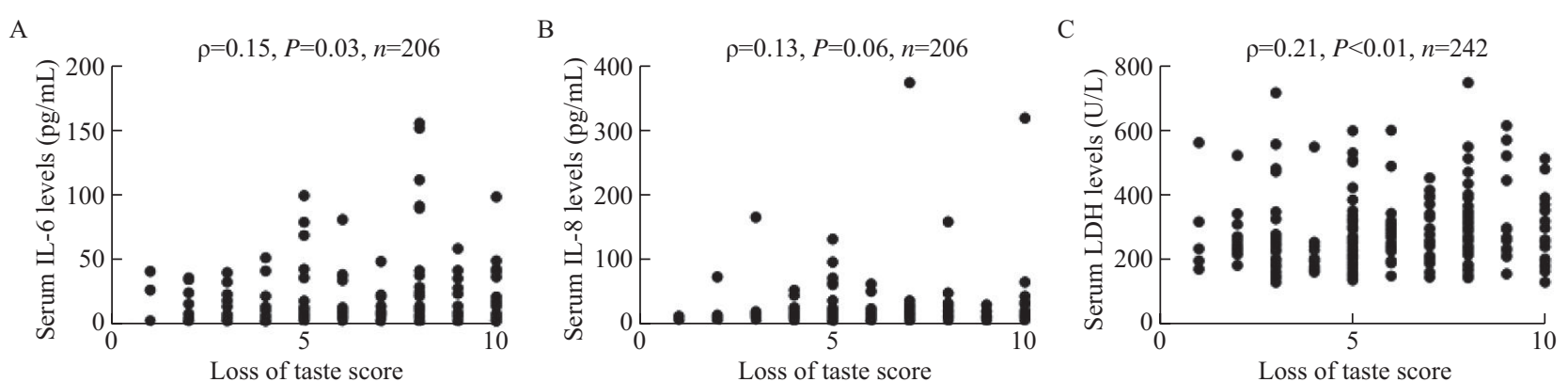

Fig. 3 Correlations between loss of taste scores and serum levels of IL-6 (A), IL-8 (B), lactose dehydrogenase (LDH) (C) Severity of self-reported loss of taste was scored by patients on a numerical rating scale of $0-10$, with 0 being "no complaint whatsoever" and 10 being "the worst imaginable complaint"

observed in our cohort ${ }^{[1,6]}$. Nevertheless, under urgent admission of COVID-19 patients, compared to systemic symptoms and comorbidities, nasal symptoms and upper airway comorbidities were very likely incompletely documented and underestimated in previous studies based on medical record analysis ${ }^{[1,12]}$. In order to overcome this limitation in real-world setting, we did recalled questionnaires by phone call to reevaluate the presence of airway comorbidities, and frequency and severity of nasal symptoms. As a result, the frequencies of COVID-19 patients with AR and CRS were 9.8\% and $6.1 \%$, respectively. Previous studies have reported that the incidence of adult AR and CRS in general population in China was $17.6 \%$ and $8 \%$, respectively, higher than that reported for COVID-19 ${ }^{[13,14]}$. Recent studies indicate that the increasing eosinophils may be an indicator of COVID-19 improvement ${ }^{[15]}$. Hence, we cannot rule out the possibility that the comorbidities of allergic and eosinophilic diseases such as AR might be potential protective factors for severe COVID-19.

Here, we found that upper respiratory tract symptoms were identified in $29.2 \%$ patients with COVID-19, with nasal obstruction, rhinorrhea, nasal itching and sneezing presenting in mild/moderate severity and loss of smell and taste presenting in moderate/severe severity. All of these upper respiratory tract symptoms have been commonly reported in other respiratory viral infection, such as influenza and rhinovirus ${ }^{[16]}$. Our data suggest that similar with the common respiratory viruses, SARS-CoV-2 may be able to infect upper respiratory tract mucosa and cause similar symptoms ${ }^{[11,17,18]}$. In addition, the result in our Wuhan cohort revealed that the self-reported symptoms of upper respiratory tract were not the first symptom in most COVID-19 patients, doubting the diagnostic value of these symptoms preceding the onset of full-blown clinical disease. However, our study only included hospitalized COVID-19 patients while the clinical manifestations of COVID-19 can range from asymptomatic infection to severe pneumonia ${ }^{[1]}$. Further investigations on non-hospitalized infected patients and patients with likely sudden onset anosmia will help to determine the role of upper airway symptoms, particular loss of smell and taste, as a screening tool for COVID-19.

Interestingly, the loss of taste and smell was the most common upper respiratory tract symptoms observed in COVID-19 patients in Wuhan, with $17.5 \%$ patients having only smell or taste disorder and $7.3 \%$ patients having both symptoms. By integrating medical record analysis and follow-up confirmation and reevaluation after discharge, we reported higher rates of smell and taste disorder in the hospitalized patients than an early report from Wuhan, which relied on medical record analysis only and had a smaller sample size ${ }^{[6]}$. However, the rates of smell and taste disorder in our cohort were significantly lower than those reported in Europe and America ${ }^{[2-5,19]}$. It may be related to the different characteristics of COVID-19 patients. In our study, asymptomatic patients and non-hospitalized patients with mild symptoms were not included. It is also likely that people with distinct ethnic/culture background may have different responses to SARS-CoV-2 infection. Previously, no large-sample-size investigation of olfactory and taste disorders in the disease caused by either SARS-CoV-1 or middle east respiratory syndrome coronavirus, that belongs to the same family of coronaviruses, was undertaken. A case report revealed the olfactory disorder in a patient infected with SARS-CoV- ${ }^{[20]}$. Nevertheless, olfactory and taste disorders are well known to be widely associated with a number of viral infections ${ }^{[21,22]}$. The frequency of patients with loss of smell in the present study was similar to that of post-viral olfactory dysfunction (PVOD) caused by the common respiratory virus, such as influenza with a rate of about $17 \%{ }^{[17,18]}$. PVOD can be caused by mechanical obstruction of odorant transmission due to edema of nasal mucosa, and/or the inflammatory impairment of the olfactory neuroepithelium and even central nervous systems ${ }^{[1,17,23]}$. Recent studies indicated that the dysfunctions of smell and taste in COVID-19 patients may share several potential mechanisms with the PVOD caused by the common respiratory virus, 
such as the inflammation and damage of the olfactory epithelium $^{[24-26]}$. However, the pathogenesis of the SARS-CoV-2-induced chemosensory dysfunctions needs to be elucidated. We found that the scores of taste and smell dysfunction showed no correlation with the scores of the other nasal symptoms including nasal obstruction, disfavoring a role of mechanical obstruction in the dysfunction of smell and taste. In fact, the symptom scores indicate that COVID-19 patients suffer from mild/moderate nasal obstruction and rhinorrhea, despite moderate/severe olfactory and taste disorder. SARS-CoV-1 has demonstrated a transneural penetration through the olfactory bulb in mice model ${ }^{[27]}$. Angiotensin converting enzyme 2, which is used by SARS-CoV-1 and SARS-CoV-2 to invade the host cells, is widely expressed on the epithelial cells in nasal and oral cavity ${ }^{[28,29]}$. This evidence suggests a neurological involvement in smell and taste disorder caused by SARS-CoV-2 infection. In this study, COVID-19 patients with severe illness had more severe taste disorder. In addition, serum levels of IL-6, a pro-inflammatory cytokine, were elevated in patients with severe taste disorder, and positively correlated with the loss of taste scores. Previous studies demonstrated that the pro-inflammatory cytokines were able to impair the function of taste buds directly, thus leading to taste dysfunction ${ }^{[30,31]}$.

The present study revealed that $95.5 \%$ patients with loss of taste and $82.1 \%$ patients with loss of smell recovered spontaneously, and most of them in 2-week time, although the severe impairment of olfactory and smell function may lead to delayed recovery. The spontaneous recovery may be a result of regeneration of the damaged olfactory epithelium and taste buds ${ }^{[32]}$. Previously, oral and topical corticosteroids have been proposed to treat viral-associated olfactory loss. However, systemic corticosteroids may impair the viral clearance. Given to the high rate of spontaneous recovery of olfactory and taste function, it seems that there is no need to use corticosteroids for treating olfactory and smell disorder in patients with COVID-19, although their administration can be continued to treat comorbidities such as AR and $\mathrm{CRS}^{[33]}$.

We have to acknowledge that there are several limitations of this study. First, the self-reported and recalled symptoms and comorbidities without diagnostic testing, especially for the symptomatic information of loss of smell and taste obtained over a month after onset, might contribute to under- or overestimation of the prevalence of these symptoms, and the strength of association with the clinical outcomes. Second, the self-report and questionnaire-based evaluation could not clearly discriminate the symptoms of loss of smell and taste in patients with COVID-19. It is the inherent limitation of the study based on selfreported data. Third, it was impossible to include the fatal cases due to the incomplete medical records regarding upper airway symptoms and comorbidities, and impossible telephone recall. Fourth, asymptomatic patients and non-hospitalized patients with mild symptoms were missed in this study. Fifth, we cannot preclude the influence of AR and CRS comorbidity on the presentation of upper airway symptoms during COVID-19. However, most of our patients claimed that their primary AR or CRS was under control at baseline and we found no difference in upper airway symptoms between patients with and without AR or CRS comorbidity (data not shown).

\section{Acknowledgments}

We thank to Cai-lin CHEN, Zhao-qin LIAN, Mengchen WANG, Jing-xian LI, Jing-xin LIU, Qiao XIAO, Si-tao HU, Chong-shu WANG, Yi-bo LIU, Jian-wen RUAN and Xue-li LI for their help with data collecting.

\section{Conflict of Interest Statement} interests.

The authors declare that they have no competing

\section{REFERENCES}

1 Guan WJ, Ni ZY, Hu Y, et al. Clinical Characteristics of Coronavirus Disease 2019 in China. N Engl J Med, 2020,382(18):1708-1720

2 Giacomelli A, Pezzati L, Conti F, et al. Self-reported Olfactory and Taste Disorders in Patients with Severe Acute Respiratory Coronavirus 2 Infection: A Crosssectional Study. Clin Infect Dis, 2020,71(15):889-890

3 Lechien JR, Chiesa-Estomba CM, De Siati DR, et al. Olfactory and gustatory dysfunctions as a clinical presentation of mild-to-moderate forms of the coronavirus disease (COVID-19): a multicenter European study. Eur Arch Otorhinolaryngol, 2020,277(8):2251-2261

4 Yan CH, Faraji F, Prajapati DP, et al. Association of chemosensory dysfunction and COVID-19 in patients presenting with influenza-like symptoms. Int Forum Allergy Rhinol, 2020,10(7):806-813

5 Roland LT, Gurrola JG, Loftus PA, et al. Smell and taste symptom-based predictive model for COVID-19 diagnosis. Int Forum Allergy Rhinol, 2020,10(7):832838

6 Mao L, Jin H, Wang M, et al. Neurologic Manifestations of Hospitalized Patients with Coronavirus Disease 2019 in Wuhan, China. JAMA Neurol, 2020,77(6):683-690

7 WHO. Clinical management of severe acute respiratory infection when novel coronavirus (2019-nCoV) infection is suspected: interim guidance. January 28, 2020 (https:// www.who.int/docs/default-source/coronaviruse/clinical -management-of-novel-cov.pdf).

8 Metlay JP, Waterer GW, Long AC, et al. Diagnosis and Treatment of Adults with Community-acquired Pneumonia. An Official Clinical Practice Guideline of the American Thoracic Society and Infectious Diseases Society of America. Am J Respir Crit Care Med, 2019, 200(7):e45-e67

9 Fokkens WJ, Lund VJ, Hopkins C, et al. European Position Paper on Rhinosinusitis and Nasal Polyps 
2020. Rhinology, 2020,58(Suppl S29):1-464

10 Zeng M, Wang H, Liao B, et al. Comparison of efficacy of fluticasone propionate versus clarithromycin for postoperative treatment of different phenotypic chronic rhinosinusitis: a randomized controlled trial. Rhinology, 2019,57(2):101-109

11 Pellegrino R, Walliczek-Dworschak U, Winter G, et al. Investigation of chemosensitivity during and after an acute cold. Int Forum Allergy Rhinol, 2017,7(2):185-91

12 Guan WJ, Liang WH, Zhao Y, et al. Comorbidity and its impact on 1590 patients with COVID-19 in China: a nationwide analysis. Eur Respir J, 2020,55(5)

13 Cheng L, Chen J, Fu Q, et al. Chinese Society of Allergy Guidelines for Diagnosis and Treatment of Allergic Rhinitis. Allergy Asthma Immunol Res, 2018,10(4):300353

14 Shi JB, Fu QL, Zhang H, et al. Epidemiology of chronic rhinosinusitis: results from a cross-sectional survey in seven Chinese cities. Allergy, 2015,70(5):533-539

15 Qin C, Zhou L, Hu Z, et al. Dysregulation of Immune Response in Patients with Coronavirus 2019 (COVID-19) in Wuhan, China. Clin Infect Dis, 2020, 71(15):762-768

16 Wang JH, Kwon HJ, Jang YJ. Detection of Parainfluenza Virus 3 in Turbinate Epithelial Cells of Postviral Olfactory Dysfunction Patients. Laryngoscope, 2007, 117(8):1445-1449

17 Suzuki M, Saito K, Min WP, et al. Identification of Viruses in Patients with Postviral Olfactory Dysfunction. The Laryngoscope, 2007,117(2):272-277

18 Malhotra P, Luka A, McWilliams CS, et al. Clinical Features of Respiratory Viral Infections Among Inpatients at a Major US Tertiary Care Hospital. South Med J, 2016,109(8):481-486

19 Luers JC, Rokohl AC, Loreck N, et al. Olfactory and Gustatory Dysfunction in Coronavirus Disease 2019 (COVID-19). Clin Infect Dis, 2020,71(16):2262-2264

20 Hwang CS. Olfactory neuropathy in severe acute respiratory syndrome: report of A case. Acta Neurol Taiwan, 2006,15(1):26-28

21 Hummel T, Landis BN, Huttenbrink KB. Smell and taste disorders. GMS Curr Top Otorhinolaryngol Head Neck Surg, 2011,10:Doc04

22 van Riel D, Verdijk R, Kuiken T. The olfactory nerve: a shortcut for influenza and other viral diseases into the central nervous system. J Pathol, 2015,235(2):277-287

23 Baig AM, Khaleeq A, Ali U, et al. Evidence of the COVID-19 Virus Targeting the CNS: Tissue Distribution, Host-Virus Interaction, and Proposed Neurotropic Mechanisms. ACS Chem Neurosci, 2020,11(7):995-998

24 Mullol J, Alobid I, Mariño-Sánchez F, et al. The Loss of Smell and Taste in the COVID-19 Outbreak: a Tale of Many Countries. Curr Allergy Asthma Rep, 2020,20(10):61

25 Butowt R, von Bartheld CS. Anosmia in COVID-19: Underlying Mechanisms and Assessment of an Olfactory Route to Brain Infection. Neuroscientist, 2020, 073858420956905

26 Cooper KW, Brann DH, Farruggia MC, et al. COVID-19 and the Chemical Senses: Supporting Players Take Center Stage. Neuron, 2020,107(2):219-233

27 Netland J, Meyerholz DK, Moore S, et al. Severe acute respiratory syndrome coronavirus infection causes neuronal death in the absence of encephalitis in mice transgenic for human ACE2. J Virol, 2008,82(15):72647275

$28 \mathrm{Xu} \mathrm{H}$, Zhong L, Deng J, et al. High expression of ACE2 receptor of 2019-nCoV on the epithelial cells of oral mucosa. Int J Oral Sci, 2020,12(1):8

$29 \mathrm{Wu}$ C, Zheng S, Chen Y, et al. Single-cell RNA expression profiling of ACE2, the putative receptor of Wuhan 2019-nCoV, in the nasal tissue. medRxiv, 2020,doi: http://doi.org/10.1101/2020.02.11.20022228

30 Kumarhia D, He L, McCluskey LP. Inflammatory stimuli acutely modulate peripheral taste function. J Neurophysiol, 2016,115(6):2964-2975

31 Henkin RI, Schmidt L, Velicu I. Interleukin 6 in Hyposmia. JAMA Otolaryngol Head Neck Surg, 2013,139(7):728-734

32 Soler ZM, Patel ZM, Turner JH, et al. A primer on viralassociated olfactory loss in the era of COVID-19. Int Forum Allergy Rhinol, 2020,10(7):814-820

33 Bousquet J, Akdis C, Jutel $\mathrm{M}$, et al. Intranasal corticosteroids in allergic rhinitis in COVID-19 infected patients: An ARIA-EAACI statement. Allergy, 2020,doi: 10.1111/011.14302

(Received Aug. 21, 2020; accepted Dec. 23, 2020) 